\title{
Direct Experimental Evidence for Strong Admixture of Different Parity States in ${ }^{11} \mathbf{L i}$
}

\author{
H. Simon, ${ }^{1}$ D. Aleksandrov, ${ }^{2}$ T. Aumann, ${ }^{3}$ L. Axelsson, ${ }^{4}$ T. Baumann, ${ }^{5}$ M. J. G. Borge, ${ }^{6}$ L. V. Chulkov, ${ }^{2}$ R. Collatz,${ }^{5}$ \\ J. Cub, ${ }^{1}$ W. Dostal, ${ }^{3}$ B. Eberlein, ${ }^{3}$ Th. W. Elze, ${ }^{7}$ H. Emling,${ }^{5}$ H. Geissel, ${ }^{5}$ A. Grünschloss, ${ }^{7}$ M. Hellström, ${ }^{5}$ \\ J. Holeczek, ${ }^{8}$ R. Holzmann, ${ }^{5}$ B. Jonson, ${ }^{4}$ J. V. Kratz, ${ }^{3}$ G. Kraus, ${ }^{5}$ R. Kulessa, ${ }^{9}$ Y. Leifels, ${ }^{5}$ A. Leistenschneider, ${ }^{7}$ \\ T. Leth, ${ }^{10}$ I. Mukha,${ }^{2}{ }^{10}$ G. Münzenberg, ${ }^{5}$ F. Nickel, ${ }^{5}$ T. Nilsson, ${ }^{11}$ G. Nyman, ${ }^{4}$ B. Petersen, ${ }^{10}$ M. Pfützner, ${ }^{5}$ \\ A. Richter, ${ }^{1}$ K. Riisager, ${ }^{10}$ C. Scheidenberger, ${ }^{5}$ G. Schrieder, ${ }^{1}$ W. Schwab, ${ }^{5}$ M. H. Smedberg, ${ }^{4}$ J. Stroth, ${ }^{7}$ A. Surowiec,${ }^{8}$ \\ O. Tengblad, ${ }^{6}$ and M. V. Zhukov ${ }^{2,4}$ \\ ${ }^{1}$ Institut für Kernphysik, Technische Universität, D-64289 Darmstadt, Germany \\ ${ }^{2}$ Kurchatov Institute, R-123182 Moscow, Russia \\ ${ }^{3}$ Institut für Kernchemie, Johannes Gutenberg-Universität, D-55099 Mainz, Germany \\ ${ }^{4}$ Fysiska Institutionen, Chalmers Tekniska Högskola och Göteborgs Universitet, S-412 96 Göteborg, Sweden \\ ${ }^{5}$ Gesellschaft für Schwerionenforschung (GSI), D-64291 Darmstadt, Germany \\ ${ }^{6}$ Institudo Estructura de la Materia, CSIC, E-28006 Madrid, Spain \\ ${ }^{7}$ Institut für Kernphysik, Johann-Wolfgang-Goethe-Universität, D-60486 Frankfurt, Germany \\ ${ }^{8}$ Institute of Physics, University of Silesia, PL-40-007 Katowice, Poland \\ ${ }^{9}$ Instytut Fizyki, Uniwersytet Jagelloński, PL-30-059 Kraków, Poland \\ ${ }^{10}$ Institut for Fysik og Astronomi, Aarhus Universitet, DK-8000 Aarhus C, Denmark \\ ${ }^{11}$ EP Division, CERN, CH-1211, Geneve 23, Switzerland
}

(Received 9 December 1998)

Peripheral fragmentation of a $287 \mathrm{MeV} /$ nucleon beam of the halo nucleus ${ }^{11} \mathrm{Li}$ incident on a carbon target has been studied in a fragment-neutron coincidence experiment. The momentum distribution of the ${ }^{10} \mathrm{Li}$ fragments gives access to the momentum distribution of the removed neutron in ${ }^{11} \mathrm{Li}$. From the shape of the distribution, the $\left(1 s_{1 / 2}\right)^{2}$ contribution to the mixture of $\left(1 s_{1 / 2}\right)^{2}$ and $\left(0 p_{1 / 2}\right)^{2}$ components in the ground-state wave function was determined to be $(45 \pm 10) \%$. The angular correlation between the knocked out neutron and the one from the decay of ${ }^{10} \mathrm{Li}$ shows a skew distribution signaling interference of these two different parity states.

PACS numbers: $27.20 .+\mathrm{n}, 24.70 .+\mathrm{s}, 25.60 . \mathrm{Gc}$

The drip line nucleus ${ }^{11} \mathrm{Li}$ is known to have a welldeveloped halo in its ground state. It was early realized [1] that the ground-state wave function has to contain a mixture of $\left(0 p_{1 / 2}\right)^{2}$ and $\left(1 s_{1 / 2}\right)^{2}$ components in order to explain the experimentally observed narrow momentum width of ${ }^{9} \mathrm{Li}$ fragments after ${ }^{11} \mathrm{Li}$ breakup reactions. Such a mixture would also explain many other experimental findings for ${ }^{11} \mathrm{Li}$ such as its large matter radius, its diluted beta-decay rate and the sizable electric dipole strength at low excitation energy (see the reviews [2-4]). Many different experiments [5-11] give indirect evidence for a $s / p$ mixture but there is, however, until now no direct determination of the $s / p$ ratio. In this paper we present new data on neutron-knockout reactions from ${ }^{11} \mathrm{Li}$ in a carbon target. These data give direct evidence for the presence of $s$ and $p$ states and allow a determination of the $s / p$ ratio.

Radioactive beams were produced in an $8 \mathrm{~g} / \mathrm{cm}^{2} \mathrm{Be}$ target by fragmentation of a primary ${ }^{18} \mathrm{O}$ beam from the heavy-ion synchrotron schwerionensynchrotron (SIS) at Gesellschaft fuer Schwerionenforschung $\mathrm{mbH}$ (GSI) and subsequently separated in the fragmentseperator (FRS) by magnetic analysis. The secondary beam of $287 \mathrm{MeV} /$ nucleon ${ }^{11} \mathrm{Li}$ was then brought to a carbon target (thickness $1.29 \mathrm{~g} / \mathrm{cm}^{2}$ ) placed in front of the ALADIN magnetic spectrometer. The neutrons, recorded in coincidence with ${ }^{9} \mathrm{Li}$, were detected in the large area neutron detector (LAND). The experimental setup was identical to the one used in experiments on ${ }^{6} \mathrm{He}$, and details about the setup can be found in Refs. $[12,13]$.

The nuclides ${ }^{6} \mathrm{He}$ and ${ }^{11} \mathrm{Li}$ may both be described as three-body systems, where none of the binary subsystems are bound. Such systems are often referred to as Borromean nuclei [14]. The main conclusion in Ref. [13], based on the ${ }^{6} \mathrm{He}$ data, is that the fragmentation process of Borromean nuclei is dominated by a sequential mechanism where one neutron is first knocked out while the rest of the system remains essentially untouched. The residual unbound two-body system subsequently decays into a neutron and a charged fragment. Such a two-step process leads to angular correlations relative to the propagation direction of the unstable fragment governed by the quantum numbers of the intermediate system. Interference effects between contributing partial waves are very important. For ${ }^{6} \mathrm{He}$, it was found [15] that a pure $\left(0 p_{3 / 2}\right)^{2}$ ground state resulted in a correlation coefficient which was a factor 2 too large while it was possible to obtain a perfect fit with a $7 \%$ admixture of the $\left(0 p_{1 / 2}\right)^{2}$ state.

In the analysis presented here we shall use the same one-neutron knockout model in the sudden approximation also for the ${ }^{11} \mathrm{Li}$ case. In spite of its simplicity and shortcomings, such a model may be justified here due 
to the loosely bound halo structure of ${ }^{11} \mathrm{Li}$ and also to the fact that the ${ }^{9} \mathrm{Li}-n$ final-state interaction cannot influence the c.m. motion of ${ }^{10} \mathrm{Li}$. We are also concerned with a light target where the Coulomb interaction is small. The experimental distributions are given in Jacobi coordinates for the three-body system $\left({ }^{9} \mathrm{Li}+n+n\right)$, defined according to Eq. (1). The initial momenta of the knocked-out and decay neutron are denoted $\mathbf{p}_{n_{1}}$ and $\mathbf{p}_{n_{2}}$, respectively. The symbol $\mathbf{p}_{f}$ is used for the ${ }^{9} \mathrm{Li}$ momentum, and $m_{n}, m_{f}$ are the neutron and the ${ }^{9} \mathrm{Li}$ masses, respectively,

$$
\left\{\begin{array}{c}
\mathbf{p}_{n f}=\frac{m_{n} m_{f}}{m_{n}+m_{f}}\left[\frac{\mathbf{p}_{n_{2}}}{m_{n}}-\frac{\mathbf{p}_{f}}{m_{f}}\right] \\
\mathbf{p}_{n(n f)}=\frac{m_{n}\left(m_{f}+m_{n}\right)}{2 m_{n}+m_{f}}\left[\frac{\mathbf{p}_{n_{1}}}{m_{n}}-\frac{\mathbf{p}_{n_{2}}+\mathbf{p}_{f}}{m_{n}+m_{f}}\right] \\
\mathbf{p}_{n_{1}}+\mathbf{p}_{n_{2}}+\mathbf{p}_{f}=0
\end{array}\right\} .
$$

Note that, by assumption, $\mathbf{p}_{n_{2}}$ and $\mathbf{p}_{f}$ are unchanged by the collision [13]. The following relation is valid in the projectile rest frame,

$$
\mathbf{p}_{n_{1}}=\mathbf{p}_{n(n f)}=-\left(\mathbf{p}_{n_{2}}+\mathbf{p}_{f}\right)=-\mathbf{p}\left({ }^{9} \mathrm{Li}+n_{2}\right) .
$$

The recoil momentum of $\mathbf{p}\left({ }^{10} \mathrm{Li}\right)=\mathbf{p}\left({ }^{9} \mathrm{Li}+n\right)$ (its transverse component, $p_{x}$, is shown in Fig. 1) therefore directly reflects the internal neutron momentum distribution in ${ }^{11} \mathrm{Li}$.

Some information about the ground-state structure may already be gained directly from the $\mathbf{p}_{f}$ and $\mathbf{p}_{n_{2}}$ distributions as demonstrated in Ref. [16]. There, the invariant mass spectrum for the $\left({ }^{9} \mathrm{Li}+n\right)$ subsystem after fragmen-

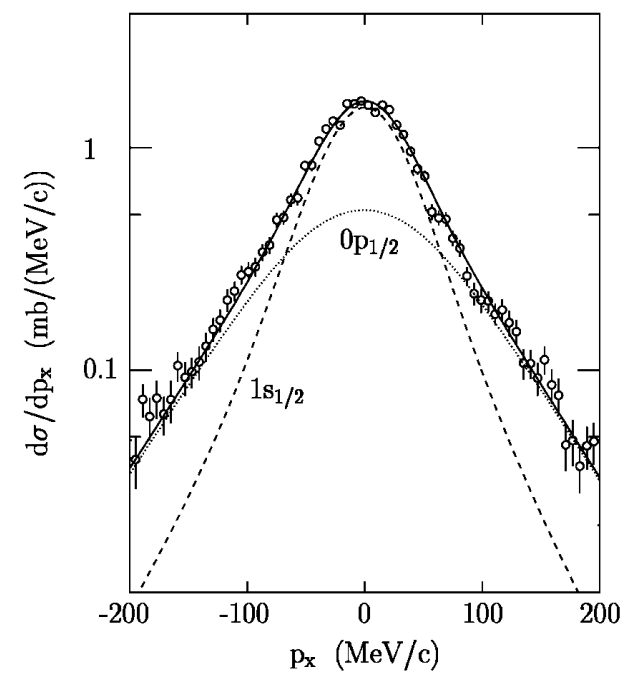

FIG. 1. Transverse momentum distribution of ${ }^{10} \mathrm{Li}$, reconstructed from the momenta of ${ }^{9} \mathrm{Li}$, and the neutron measured in coincidence after neutron knockout from ${ }^{11} \mathrm{Li}(C$ target). Dashed and dotted lines represent $\left(1 s_{1 / 2}\right)^{2}$ and $\left(0 p_{1 / 2}\right)^{2}$ singleparticle momentum distributions calculated for one of the halo neutrons in ${ }^{11} \mathrm{Li}$, including core shadowing and the experimental resolution. The solid line represents the best fit to the data obtained with a $45 \%\left(1 s_{1 / 2}\right)^{2}$ contribution. tation of ${ }^{11} \mathrm{Li}$ was extracted. Two low-lying structures of comparable weights were observed and interpreted as $s$ and $p$ states in ${ }^{10} \mathrm{Li}$, where the lowest part corresponds to the $s$-wave decay.

It has recently been shown [17-19] that the condition of fragment survival gives a momentum distribution which is mainly determined by the asymptotic part of the halo-neutron wave function. This allows for a quantitative determination of the $s$-wave admixture in the ${ }^{11} \mathrm{Li}$ ground state directly from the ${ }^{10} \mathrm{Li}$ momentum distribution. The data displayed in Fig. 1 were fitted using the first spherical Hankel functions for the $s$ and $p$ neutrons using the formulas given in Ref. [17]. Following the prescription therein the requirement of fragment survival was imitated by implying a cylindrical cut, $R_{\text {cut }}$, over the distance between neutron and core. $R_{\text {cut }}$ was used as a free parameter in the fit, and the calculated distribution was folded with the experimental resolution. The result displayed in Fig. 1, obtained under the assumption of $s$ and $p$ components only, gives a $(45 \pm 10) \%$ admixture of $\left(1 s_{1 / 2}\right)^{2}$ in the ${ }^{11} \mathrm{Li}$ ground state. The allowed region for $R_{\text {cut }}$ is $1.8<R_{\text {cut }}<$ $3.0 \mathrm{fm}$. Values below the lower limit fail to reproduce the central part of the distribution while values above the upper limit cannot reproduce its wings.

The presence of angular correlations can be tested by comparing the standard deviations $\sigma$ of the transverse momentum distributions for core fragments $\overline{p_{x}^{2}}(f)$, the spectator neutron $\overline{p_{x}^{2}}(n)$, and their center-of-mass movement $\overline{p_{x}^{2}}(f+n)[20]$ using

$$
\begin{aligned}
\overline{p_{x}^{2}}(f+n) & =\overline{p_{x}^{2}}(n)+\overline{p_{x}^{2}}(f)+2 \overline{p_{x}(n) p_{x}(f)} \\
& =\overline{p_{x x}^{2}}(n)+\overline{p_{x}^{2}}(f)+2 \cos z \theta \sqrt{\overline{p_{x}^{2}}(n) \overline{p_{x}^{2}}(f)} .
\end{aligned}
$$

For simplicity, we use Gaussian fits to the distributions in this analysis which give the following $\sigma$ values:

$$
\sigma_{{ }^{10} \mathrm{Li}}=23.8(4) \sigma^{9} \mathrm{Li}=23.5(4) \sigma_{n}=15.0(3)(\mathrm{MeV} / c)
$$

which, with Eq. (3), result in $\theta=107(2)^{\circ}$ (see inset of Fig. 2). The values in brackets are the statistical errors. The correlation term is thus negative and nonzero. By using Eq. (1), one can also estimate that the angle $\theta_{n f}$, between the vectors $\mathbf{p}\left({ }^{9} \mathrm{Li}+n\right)$ and $\mathbf{p}_{n f}$, is equal to $101\left(2^{\circ}\right.$ ) (see inset of Fig. 2). This indicates that, contrary to the ${ }^{6} \mathrm{He}$ case where the mean value of this angle is equal to $90^{\circ}$ [12], the angular distribution is asymmetric, signaling contributions from orbitals with different parities. Moreover, the neutron spectator moves preferably in the direction of the participant neutron which indicates a $n-n$ correlation.

The measured distribution of $\theta_{n f}$, the angle between the two vectors $\mathbf{p}\left({ }^{9} \mathrm{Li}+n\right)$ and $\mathbf{p}_{n f}$, is shown in Fig. 2 as open circles with error bars. This distribution is influenced by the experimental resolution and the finite acceptance of the setup. Still, the skew distribution provides 


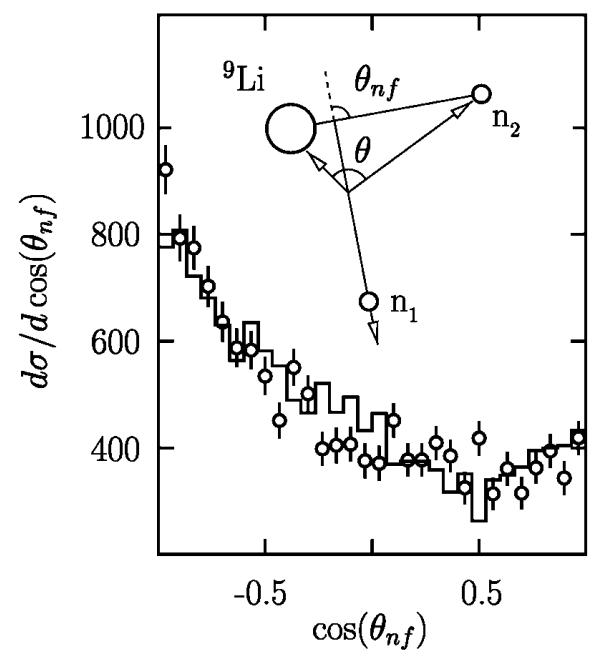

FIG. 2. Distribution of the decay neutrons from ${ }^{10} \mathrm{Li}$ formed in ${ }^{11} \mathrm{Li}$ neutron knockout reactions. The inset shows a schematic diagram of the reaction where $\theta_{n f}$ is the angle between the momentum direction of ${ }^{10} \mathrm{Li}$ reconstructed as a sum of the momenta of the ${ }^{9} \mathrm{Li}$ fragment and the decay neutronand the direction of the $n+{ }^{9} \mathrm{Li}$ relative momentum $\mathbf{p}_{n f}$. The distribution asymmetry can be explained only if one assumes contributions from interfering $s$ and $p$ states in ${ }^{10} \mathrm{Li}$.

direct evidence for a strong mixture of different parity states since it implies that the distribution must contain a linear term in $\cos \left(\theta_{n f}\right)$. This is model independent proof of the appearance of the $s$ and $p$ states in ${ }^{11} \mathrm{Li}$. The original distribution was restored by a Monte Carlo method which takes into account all the basic parameters of the setup, and an iteration procedure was used to fit the experimental data. The histogram in Fig. 2 is the result of such a procedure.

The undisturbed distribution used in the Monte Carlo calculations was approximated by a polynomial expansion in terms of $\cos \left(\theta_{n f}\right)$ :

$$
\mathcal{W}\left(\theta_{n f}\right)=1-1.03(4) \cos \left(\theta_{n f}\right)+1.41(8) \cos ^{2}\left(\theta_{n f}\right) \text {. }
$$

This gives a mean value of $\theta_{n f}=103.4(2.1)^{\circ}$, in agreement with the above estimate.

We now proceed to investigate how Eq. (4) is related to the $s$ and $p$ components in the ${ }^{11} \mathrm{Li}$ ground-state wave function. For this, we restrict ourselves to considering only three different spin and angular momentum configurations in ${ }^{11} \mathrm{Li}$, neglecting for simplicity the spin of the ${ }^{9} \mathrm{Li}$ core, namely, $\left(S=0, L=\ell_{x}=\ell_{y}=0\right),(S=$ $\left.0, L=0, \ell_{x}=\ell_{y}=1\right)$, and $\left(S=1, L=\ell_{x}=\ell_{y}=1\right)$ [21] and the corresponding amplitude factors $A_{\ell_{x} \ell_{y}}^{\mathrm{SL}}$. The first two components, $A_{00}^{00}$ and $A_{11}^{00}$, will lead to the interference term in the angular correlation function. It should be noted that in general these amplitudes are complex for the decaying states and can be written as $\left|A_{\ell_{x} \ell_{y}}^{\mathrm{SL}}\right| e^{i \delta_{\ell_{x} \ell_{y}}^{\mathrm{SL}}}$. Consequently, only one phase parameter $\phi_{\text {rel }}=\delta_{00}^{00}-\delta_{11}^{00}$ enters into the final expression for the angular correlation. The assumption of a sudden removal of the knocked-out neutron means that one, in the first approximation, can consider the moduli of these amplitudes $\left|A_{\ell_{x} \ell_{y}}^{\mathrm{SL}}\right|$ to correspond to those of the ${ }^{11} \mathrm{Li}$ ground state. This should hold provided that shadowing effects are small which is actually the case since the $s / p$ ratio is stable over a large range of $R_{\text {cut }}$ values as mentioned above. The representation of the spin-angular part of the $\left({ }^{9} \mathrm{Li}+n+n\right)$ wave function (in the continuum) may then be written in $j j$ coupling as [22]

$$
\begin{aligned}
\psi= & A_{00}^{00}\left[s_{1 / 2} s_{1 / 2}\right]_{0} \\
& +A_{11}^{00}\left(\sqrt{\frac{1}{3}}\left[p_{1 / 2} p_{1 / 2}\right]_{0}+\sqrt{\frac{2}{3}}\left[p_{3 / 2} p_{3 / 2}\right]_{0}\right) \\
& +A_{11}^{11}\left(\sqrt{\frac{2}{3}}\left[p_{1 / 2} p_{1 / 2}\right]_{0}-\sqrt{\frac{1}{3}}\left[p_{3 / 2} p_{3 / 2}\right]_{0}\right) .
\end{aligned}
$$

Similar to the ${ }^{6} \mathrm{He}$ case [12], we shall further assume that $\mathcal{W}\left(\theta_{n f}\right) \propto|\psi|^{2}$. We can then determine all of the parameters $\left|A_{00}^{00}\right|^{2},\left|A_{11}^{00}\right|^{2},\left|A_{11}^{11}\right|^{2}$, and $\phi_{\text {rel }}$ from a fit to our experimental angular distribution. The system is underdetermined since we have four free parameters while the fit to the polynomial only needs three. The main aim here is, however, to determine the relative weights of the $\left(1 s_{1 / 2}\right)^{2}$ and $\left(0 p_{1 / 2}\right)^{2}$ components. We vary $\phi_{\text {rel }}$ in a region where $\mathcal{W}\left(\theta_{n f}\right)$ gives a reasonable fit to the data in Fig. 2 which gives $0^{\circ}<\phi_{\text {rel }}<60^{\circ}$. The result is shown in Fig. 3. For $\phi_{\text {rel }}>60^{\circ}$ the fit becomes unstable. The $\left(1 s_{1 / 2}\right)^{2}$ contribution is shown in Fig. 3 as a solid

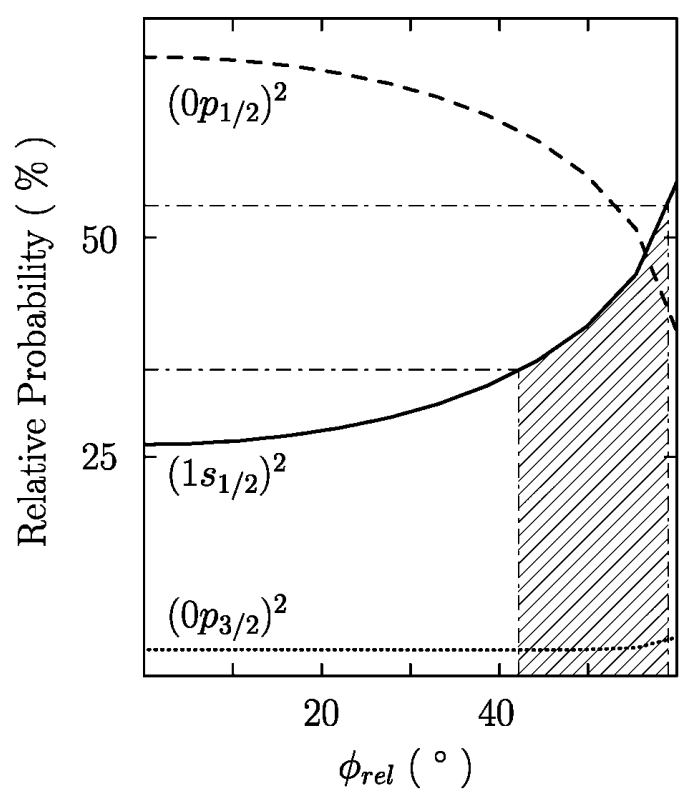

FIG. 3. Relative probabilities of the $s$ and $p$ components in the ${ }^{11} \mathrm{Li}$ halo wave function versus the phase $\phi_{\text {rel }}$ between the interfering $s$ and $p$ waves in the decaying ${ }^{10} \mathrm{Li}$ obtained from a fit to the experimental angular distributions shown in Fig. 2. The shaded area shows possible values for the relative phase within the uncertainties in the determination of the $s$ to $p$ ratio. 
line and varies between $25 \%$ and $55 \%$ while the $\left(p_{3 / 2}\right)^{2}$ contribution (shown as a dotted line) gives a maximal relative weight of $(3-5) \%$ in the total wave function. The observed small value is in good agreement with the shell-model expectation [22] that the $p_{3 / 2}$ state is already occupied in ${ }^{9} \mathrm{Li}$. We can now proceed and use our result for the $\left(1 s_{1 / 2}\right)^{2}$ contribution from Fig. 1 to give the range of values for $\phi_{\text {rel }}$. This is shown as the shaded area in Fig. 3 giving $42^{\circ}<\phi_{\text {rel }}<59^{\circ}$.

The quantitative and qualitative analysis of the breakup data from ${ }^{11} \mathrm{Li}$ in a carbon target, that has been performed within the framework of our model, can be summarized as follows.

(i) The observed strong asymmetry in the angular correlation function gives a direct and model independent indication of an admixture of states with different parity in the ground state of ${ }^{11} \mathrm{Li}$. Within the framework of recent experimental and theoretical knowledge, they can be understood as $p_{1 / 2}$ and $s_{1 / 2}$ states in the ${ }^{9} \mathrm{Li}+n$ subsystem.

(ii) The analysis of the invariant-mass spectrum in the $\left({ }^{9} \mathrm{Li}+n\right)$ subsystem and the transverse momentum distribution of the unstable ${ }^{10} \mathrm{Li}$ fragments show contributions from both $s$ and $p$ shell neutrons. The contribution of the $\left(1 s_{1 / 2}\right)^{2}$ state in the ${ }^{11} \mathrm{Li}$ ground-state wave function is determined to be $(45 \pm 10) \%$.

(iii) Some indication of weak $n-n$ correlations is found from a comparison of the standard deviations of the momentum distributions for the ${ }^{9} \mathrm{Li}$ fragment and the decay neutron.

(iv) We may speculate that the relative phase shift might be connected with final-state interactions in the $\left({ }^{9} \mathrm{Li}+n\right)$ subsystem or with a shadow effect during the knock-out process. For the ${ }^{6} \mathrm{He}$ case [15], the corresponding phase shift was found to be very close to the experimental one, between $p_{3 / 2}$ and $p_{1 / 2}$ waves in $\alpha+n$ scattering at the energy corresponding to the ${ }^{5} \mathrm{He}$ ground state.

This work was supported by the BMBF under Contracts No. 06 DA 820, No. 06 OF 112, and No. 06 MZ 476, and by GSI via Hochschulzusammenarbeitsvereinbarungen under Contracts No. DA RICK, No. OF ELZ, and No. MZ KRK, and partly supported by the Polish Committee of Scientific Research under Contract No. PBZ/ PB03/113/09, EC under Contract No. ERBCHGE-CT920003, CICYT under Contract No. AEN92-0788-C02-02
(M. J. G. B.), and WTZ under Contract No. RUS 609-96. One of us (B. J.) acknowledges support from an Alexander von Humboldt Research Award.

[1] I. J. Thomson and M. V. Zhukov, Phys. Rev. C 49, 1904 (1994).

[2] P. G. Hansen, A. S. Jensen, and B. Jonson, Annu. Rev. Nucl. Part. Sci. 45, 591 (1995).

[3] I. Tanihata, J. Phys. G 22, 157 (1996).

[4] B. Jonson and K. Riisager, Philos. Trans. R. Soc. London A 356, 2063 (1998).

[5] B. M. Young et al., Phys. Rev. C 49, 279 (1994).

[6] M. Zinser et al., Phys. Rev. Lett. 75, 1719 (1995).

[7] R. A. Kryger et al., Phys. Rev. C 47, R2439 (1993).

[8] M. J. G. Borge et al., Phys. Rev. C 55, R8 (1997).

[9] D. J. Morrissey et al., Nucl. Phys. A627, 22 (1997).

[10] N. Aoi et al., Nucl. Phys. A 616, 181c (1997).

[11] T. Teranishi et al., Phys. Lett. B 407, 110 (1997).

[12] L. V. Chulkov et al., Phys. Rev. Lett. 79, 201 (1997).

[13] D. Aleksandrov et al., Nucl. Phys. A633, 234 (1998).

[14] M. V. Zhukov et al., Phys. Rep. 231, 151 (1993).

[15] L. V. Chulkov and G. Schrieder, Z. Phys. A 359, 231 (1997).

[16] M. Zinser et al., Nucl. Phys. A619, 151 (1997).

[17] P. G. Hansen, Phys. Rev. Lett. 77, 1016 (1996).

[18] K. Henken et al., Phys. Rev. C 54, 3043 (1996).

[19] T. Aumann et al., Nucl. Phys. A640, 24 (1998).

[20] This method is based on the fact obtained in the present experiment that the fragment momentum distributions from ${ }^{11} \mathrm{Li}$ are spherically symmetric so that the momentum distributions on each of three Cartesian coordinates are almost identical. One can therefore perform the analysis on one axis only.

[21] Here $S$ denotes the total spin of the two-neutron subsystem which can be either in singlet state $S=0$ or in triplet state $S=1, \ell_{x}$ and $\ell_{y}$ are the angular momenta conjugated to the Jacobi coordinates $\mathbf{p}_{n f}$ and $\mathbf{p}_{n(n f)}$, respectively, and $L$ is the sum $\vec{\ell}_{x}+\vec{\ell}_{y}$.

[22] Note that the $p_{3 / 2}$ components of the valence neutroncore motion are included explicitly in the expression for $\psi$. These states are, however, already occupied in the ${ }^{9} \mathrm{Li}$ core, which means that only a small contribution from them to the total $\psi$ is expected. This is, as can be seen, verified in the fit of the data. 